\title{
TRANSFORMATION FORMULA OF THE “2ND” ORDER MOCK THETA FUNCTION
}

\author{
KAZUHIRO HIKAMI
}

ABstract. We give a transformation formula for the " 2 nd order" mock theta function

$$
D_{5}(q)=\sum_{n=0}^{\infty} \frac{(-q)_{n}}{\left(q ; q^{2}\right)_{n+1}} q^{n}
$$

which was recently proposed in connection with the quantum invariant for the Seifert manifold.

\section{Introduction and Statement of Results}

We study the transformation formula for the $q$-series $D_{5}(q)$ defined by

$$
\begin{aligned}
D_{5}(q) & =\sum_{n=0}^{\infty} \frac{(-q)_{n}}{\left(q ; q^{2}\right)_{n+1}} q^{n} \\
& =1+2 q+4 q^{2}+6 q^{3}+10 q^{4}+16 q^{5}+23 q^{6}+\cdots
\end{aligned}
$$

where as usual we mean $q=\exp (2 \pi \mathrm{i} \tau)$ with $\tau \in \mathbb{H}$. This $q$-series can be rewritten as

$$
D_{5}(q)=\frac{1}{\left[\left(q ; q^{2}\right)_{\infty}\right]^{2}} \sum_{n=0}^{\infty}\left[\left(q ; q^{2}\right)_{n}\right]^{2} q^{2 n}
$$

which can be proved by setting $\alpha=q, \beta=q, \gamma=0, z=q^{2}$ in the following transformation formula of the $q$-hypergeometric functions (see, e.g., Ref. 1)

$$
\sum_{n=0}^{\infty} \frac{\left(\alpha ; q^{2}\right)_{n}(\beta)_{2 n}}{\left(q^{2} ; q^{2}\right)_{n}(\gamma)_{2 n}} z^{n}=\frac{(\beta)_{\infty}\left(\alpha z ; q^{2}\right)_{\infty}}{(\gamma)_{\infty}\left(z ; q^{2}\right)_{\infty}} \sum_{m=0}^{\infty} \frac{\left(\frac{\gamma}{\beta}\right)_{m}\left(z ; q^{2}\right)_{m}}{(q)_{m}\left(\alpha z ; q^{2}\right)_{m}} \beta^{m} .
$$

The $q$-series $D_{5}(q)$ was introduced in Ref. 7 in connection with the quantum invariant of 3manifold. The definition (1) can be extended to $|q|>1$, and we can introduce a new $q$-series by replacing $q$ with $1 / q$ in the summand of (11);

$$
\begin{aligned}
D_{5}^{*}(q) & =\sum_{n=0}^{\infty}(-1)^{n} q^{n(n+1) / 2} \frac{(-q)_{n}}{\left(q ; q^{2}\right)_{n+1}} \\
& =1-q^{2}+q^{6}-q^{12}+q^{20}-q^{30}+q^{42}+\cdots
\end{aligned}
$$

Date: September 21, 2005. Revised on October 24, 2005.

2000 Mathematics Subject Classification. 33D15, 11F27, 57M27.

Key words and phrases. Mock Theta Function, Quantum Invariant, Seifert Manifold, $q$-Hypergeometric Function . 
A limiting value of $D_{5}^{*}(q)$ when $q$ is the root of unity is related to the $\mathrm{SU}(2)$ Witten-ReshetikhinTuraev (WRT) invariant $\tau_{N}(\mathcal{M})[13,15]$ for 3 -manifold $\mathcal{M}$. Precisely, we have [6,7]

$$
\left(\mathrm{e}^{2 \pi \mathrm{i} / N}-1\right) \cdot \tau_{N}(M(2,2,2))=2\left(1-2 D_{5}^{*}\left(\mathrm{e}^{\pi \mathrm{i} / N}\right)\right)
$$

Here $M(2,2,2)$ denotes the Seifert prism manifold $O o 0(-1 ;(2,1),(2,1),(2,1))$ (see, e.g., Refs. 9 , $10)$.

Lawrence and Zagier pointed out [8] that the Eichler integral of the modular form with weight $3 / 2$ has a nearly modular property, and that the WRT invariant for the Poincaré homology sphere is regarded as a limiting value of the Eichler integral. This result is further extended to the WRT invariant for other Seifert fibered manifolds, and especially it was shown that the WRT invariant (5) for the prism manifold $M(2,2,2)$ has a nearly modular property under a transformation $1 / N \leftrightarrow$ $-N[6]$.

Well known is that the Ramanujan mock theta functions satisfy this type of transformation formula $[12,14]$. It is demonstrated in Refs. 7, 8 that the Eichler integral which reduces to the WRT invariant for the Poincaré homology sphere gives the 5th order Ramanujan mock theta function when we replace $q$ by $1 / q$. Therefore the $q$-series $D_{5}(q)$ defined in (1) is expected to be a mock theta function à la Ramanujan. In this short note, we shall give the transformation formula for the function $D_{5}(q)$, and we prove that the function is indeed the mock theta function.

Among Ramanujan's mock theta functions, we recall the third order mock theta functions [14] defined by

$$
\begin{aligned}
\omega(q) & =\sum_{n=0}^{\infty} \frac{q^{2 n(n+1)}}{\left[\left(q ; q^{2}\right)_{n+1}\right]^{2}} \\
& =\sum_{n=0}^{\infty} \frac{q^{n}}{\left(q ; q^{2}\right)_{n+1}} \\
f(q) & =\sum_{n=0}^{\infty} \frac{q^{n^{2}}}{\left[(-q)_{n}\right]^{2}} \\
& =2-\sum_{n=0}^{\infty}(-1)^{n} \frac{q^{n}}{(-q)_{n}}
\end{aligned}
$$

Here the second equalities are proved in Ref. 4. As was proved in Ref. 14, these functions can be written in the form of the Lerche sum;

$$
\begin{gathered}
\left(q^{2} ; q^{2}\right)_{\infty} \cdot \omega(q)=\sum_{n \in \mathbb{Z}}(-1)^{n} \frac{q^{3 n(n+1)}}{1-q^{2 n+1}} \\
(q)_{\infty} \cdot f(q)=2 \sum_{n \in \mathbb{Z}}(-1)^{n} \frac{q^{\frac{1}{2} n(3 n+1)}}{1+q^{n}}
\end{gathered}
$$


To study the transformation formula for $D_{5}(q)$, we introduce two functions;

$$
\begin{aligned}
h_{1}(q) & =\sum_{n=0}^{\infty} \frac{(-q)_{2 n}}{\left[\left(q ; q^{2}\right)_{n+1}\right]^{2}} q^{n} \\
& =1+3 q+7 q^{2}+14 q^{3}+27 q^{4}+49 q^{5}+84 q^{6}+\cdots \\
h_{2}(q) & =\sum_{n=0}^{\infty}(-1)^{n} \frac{\left(q ; q^{2}\right)_{n}}{\left[\left(-q^{2} ; q^{2}\right)_{n}\right]^{2}} q^{n^{2}} \\
& =1-q+q^{2}+2 q^{3}-q^{4}-4 q^{5}+q^{6}+\cdots
\end{aligned}
$$

As was shown in Ref. 3 as Entry 12.3.9 and Entry 12.2.1 which originally appeared in Ref. 12, those functions can also be rewritten as the form of the Lerche sum as follows;

$$
\begin{gathered}
h_{1}(q)=\frac{1}{2} \frac{(-q)_{\infty}}{(q)_{\infty}} \sum_{n \in \mathbb{Z}}(-1)^{n} \frac{q^{n(n+2)}}{1-q^{2 n+1}} \\
h_{2}(q)=\frac{(q)_{\infty}}{\left[\left(q^{2} ; q^{2}\right)_{\infty}\right]^{2}} \sum_{n \in \mathbb{Z}} \frac{q^{\frac{1}{2} n(n+1)}}{1+q^{n}}+\frac{1}{2} \frac{\left[(q)_{\infty}\right]^{5}}{\left[\left(q^{2} ; q^{2}\right)_{\infty}\right]^{4}}
\end{gathered}
$$

As Entry 12.4.5 in Ref. 3 proves

$$
D_{5}(q)=2 h_{1}(q)-\left[\frac{\left(q^{2} ; q^{2}\right)_{\infty}}{(q)_{\infty}}\right]^{2} \omega(q)
$$

we need to study the transformation formulae for the functions $h_{1}(q)$ and the third order mock theta function $\omega(q)$ defined in (6).

In Ref. 14 Watson derived the following transformation of the third order mock theta functions, $\omega(q)$ and $f(q)$;

$$
\int_{0}^{\infty} \mathrm{e}^{-\frac{3}{4} \alpha x^{2}} \frac{\cosh (\alpha x / 2)}{\cosh (3 \alpha x / 2)} \mathrm{d} x=-\sqrt{\frac{4 \pi}{3 \alpha}} q^{2 / 3} \omega(q)+\frac{1}{\sqrt{3}} \frac{\pi}{\alpha} q_{1}^{-1 / 12} f\left(q_{1}^{2}\right)
$$

where we have set

$$
q=\mathrm{e}^{-\alpha} \quad q_{1}=\mathrm{e}^{-\pi^{2} / \alpha}
$$

with $\mathfrak{R} \alpha>0$.

As will be proved in Section 2 , we have the following transformation formula for our functions $h_{1}(q)$ and $h_{2}(q)$

Theorem 1. Let the functions $h_{1}(q)$ and $h_{2}(q)$ be defined in (14) and (15) respectively. We have

$$
\int_{-\infty}^{\infty} \frac{\mathrm{e}^{-\alpha x^{2}}}{\cosh (\alpha x)} \mathrm{d} x=-4 \sqrt{\frac{\pi}{\alpha}} q^{3 / 4} h_{1}(q)+\frac{\pi}{\alpha} q_{1}^{-1 / 4}\left(h_{2}\left(q_{1}^{2}\right)-\frac{1}{2} \frac{\left[\left(q_{1}^{2} ; q_{1}^{2}\right)_{\infty}\right]^{5}}{\left[\left(q_{1}^{4} ; q_{1}^{4}\right)_{\infty}\right]^{4}}\right)
$$

where we have set parameters $q$ and $q_{1}$ as in (18) with $\mathfrak{R} \alpha>0$. 
The left hand side of (19) is the Mordell integral, and the properties of this integral was studied in detail in Ref. 11 (see also Refs. 2,5).

This theorem proves that the function $D_{5}(q)$ can be written as a sum of two mock theta functions, $h_{1}(q)$ and $\omega(q)$, as in (16). Thus we can conclude that the $q$-series $D_{5}(q)$, which was originally introduced based on studies of the WRT invariant, is indeed the mock theta function, and that the transformation formula of $D_{5}(q)$ is given by combining (16), (17), and (19).

\section{Proof of Theorem 1}

Both the integral in the left hand side and the $q$-series in the right hand side (see definitions (12) and (13) ) of (19) are analytic in $\mathfrak{R} \alpha>0$. Accordingly we can assume $\alpha \in \mathbb{R}_{>0}$ to simplify our proof thanks to the analytic continuation.

We study the integral defined by

$$
K=\frac{1}{2 \pi \mathrm{i}} \int_{C} \frac{\pi}{\sin (\pi z)} \frac{\mathrm{e}^{-\alpha z(z+2)}}{1-\mathrm{e}^{-\alpha(2 z+1)}} \mathrm{d} z
$$

where a contour $C$ encircles a real axis counterclockwise. As the integrand converges to zero in $\mathfrak{R} z \rightarrow \pm \infty$, the integral $K$ can be decomposed into

$$
K=\frac{1}{2 \pi \mathrm{i}}\left(\int_{-\infty-\mathrm{i} 0}^{\infty-\mathrm{i} 0}+\int_{\infty+\mathrm{i} 0}^{-\infty+\mathrm{i} 0}\right) \frac{\pi}{\sin (\pi z)} \frac{\mathrm{e}^{-\alpha z(z+2)}}{1-\mathrm{e}^{-\alpha(2 z+1)}} \mathrm{d} z
$$

which we set $K=K_{1}+K_{2}$. It is easy to see that, by the Cauchy theorem, the integral $K$ (20) is given by

$$
K=\sum_{n \in \mathbb{Z}}(-1)^{n} \frac{q^{n(n+2)}}{1-q^{2 n+1}}-\frac{\pi}{2 \alpha} q^{-3 / 4}
$$

We first consider the integral $K_{2}$. On the path contour of $K_{2}$ which is in the upper half plane we can apply the Fourier expansion

$$
\frac{1}{\sin (\pi z)}=-2 \mathrm{i} \sum_{n=0}^{\infty} \mathrm{e}^{(2 n+1) \pi \mathrm{i} z}
$$

and substituting this expression, we obtain

$$
K_{2}=\frac{1}{2 \pi \mathrm{i}} \sum_{n=0}^{\infty} \int_{-\infty+\mathrm{i} 0}^{\infty+\mathrm{i} 0} F_{n}(z) \mathrm{d} z
$$

where the integrand $F_{n}(z)$ is defined by

$$
F_{n}(z)=2 \pi \mathrm{i} \frac{\mathrm{e}^{(2 n+1) \pi \mathrm{i} z-\alpha z^{2}-2 \alpha z}}{1-\mathrm{e}^{-\alpha(2 z+1)}}
$$

We see that the integrand $F_{n}(z)$ has simple poles at

$$
z=\frac{m}{\alpha} \pi \mathrm{i}-\frac{1}{2} \equiv z_{m}
$$


for $m \in \mathbb{Z}$, and the residues of $F_{n}(z)$ at $z=z_{m}$ are computed to be

$$
\lambda_{n, m}=\frac{\pi \mathrm{i}}{\alpha} \mathrm{e}^{(2 n+1) z_{m} \pi \mathrm{i}-\alpha z_{m}\left(z_{m}+2\right)}
$$

Then we obtain

$$
\begin{aligned}
\frac{1}{2 \pi \mathrm{i}} & \sum_{n=0}^{\infty}\left(\int_{-\infty+\mathrm{i} 0}^{\infty+\mathrm{i} 0}-\int_{-\infty+\frac{2 n+1}{2 \alpha} \pi \mathrm{i}}^{\infty+\frac{2 n+1}{2 \alpha} \pi \mathrm{i}}\right) F_{n}(z) \mathrm{d} z \\
& =\sum_{n=1}^{\infty}\left(\lambda_{n, 1}+\lambda_{n, 2}+\cdots+\lambda_{n, n}\right) \\
& =\sum_{m=1}^{\infty}\left(\lambda_{m, m}+\lambda_{m+1, m}+\cdots\right) \\
& =\sum_{m=1}^{\infty} \lambda_{m, m} \frac{1}{1-\mathrm{e}^{2 \pi \mathrm{i} z m}} \\
& =\frac{\pi}{\alpha} \mathrm{e}^{\frac{3}{4} \alpha} \sum_{m=1}^{\infty} \frac{q_{1}^{m(m+1)}}{1+q_{1}^{2 m}}
\end{aligned}
$$

On the other hand, we have

$$
\begin{aligned}
\frac{1}{2 \pi \mathrm{i}} \int_{-\infty+\frac{2 n+1}{2 \alpha} \pi \mathrm{i}}^{\infty+\frac{2 n+1}{2 \alpha} \pi \mathrm{i}} F_{n}(z) \mathrm{d} z & =\int_{-\infty}^{\infty} F_{n}\left(x+\frac{2 n+1}{2 \alpha} \pi \mathrm{i}-\frac{1}{2}\right) \mathrm{d} x \\
& =-\pi \mathrm{i} q^{-3 / 4} q_{1}^{(2 n+1)^{2} / 4} \int_{-\infty}^{\infty} \frac{\mathrm{e}^{-\alpha x^{2}}}{\cosh (\alpha x)} \mathrm{d} x
\end{aligned}
$$

Combining these results, we obtain

$$
K_{2}=\frac{\pi}{\alpha} q^{-3 / 4} \sum_{m=1}^{\infty} \frac{q_{1}^{m(m+1)}}{1+q_{1}^{2 m}}-\frac{1}{2} q^{-3 / 4}\left(\sum_{n=0}^{\infty} q_{1}^{(2 n+1)^{2} / 4}\right) \int_{-\infty}^{\infty} \frac{\mathrm{e}^{-\alpha x^{2}}}{\cosh (\alpha x)} \mathrm{d} x
$$

Integral $K_{1}$ from the lower half plane can be computed in the same manner. Applying the Jacobi triple product formula, we finally obtain the transformation formula (19) which proves Theorem 1 after the analytic continuation.

\section{ACKNOWLedgments}

This work is supported in part by the Grant-in-Aid for Young Scientists from the Ministry of Education, Culture, Sports, Science and Technology of Japan.

\section{References}

[1] G. E. Andrews, q-identities of Auluck, Carlitz, and Rogers, Duke Math. J. 33, 575-582 (1966).

[2] — Mordell integrals and Ramanujan's “lost” notebook, in M. I. Knopp, ed., Analytic Number Theory, Lecture Notes in Math. 899, pp. 10-48, Springer, New York, 1981.

[3] G. E. Andrews and B. C. Berndt, Ramanujan's Lost Notebook part I, Springer, New York, 2005. 
[4] N. J. Fine, Basic Hypergeometric Series and Applications, no. 27 in Math. Surv. Monographs, AMS, Providence, 1988.

[5] G. H. Hardy, P. V. S. Aiyar, and B. M. Wilson, eds., Collected papers of Srinivasa Ramanujan, Amer. Math. Soc., Providence, 2000.

[6] K. Hikami, On the quantum invariant for the spherical Seifert manifold, preprint (2005), math-ph/0504082.

[7] - Mock (false) theta functions as quantum invariants, preprint (2005), math-ph/0506073.

[8] R. Lawrence and D. Zagier, Modular forms and quantum invariants of 3-manifolds, Asian J. Math. 3, 93-107 (1999).

[9] J. Milnor, On the 3-dimensional Brieskorn manifolds $M(p, q, r)$, in L. P. Neuwirth, ed., Knots, Groups, and 3-Manifolds, pp. 175-225, Princeton Univ. Press, 1975, papers Dedicated to the Memory of R. H. Fox.

[10] J. M. Montesinos, Classical Tessellations and Three-Manifolds, Springer-Verlag, Berlin, 1987.

[11] L. J. Mordell, The definite integral $\int_{-\infty}^{\infty} \frac{e^{a x^{2}+b x}}{e^{c x}+d} d x$ and the analytic theory of numbers, Acta Math. 61, 323-360 (1933).

[12] S. Ramanujan, The Lost Notebook and other unpublished papers, Narosa, New Delhi, 1987.

[13] N. Yu. Reshetikhin and V. G. Turaev, Invariants of 3-manifolds via link polynomials and quantum groups, Invent. Math. 103, 547-597 (1991).

[14] G. N. Watson, The final problem: an account of the mock theta functions, J. London Math. Soc. 11, 55-80 (1936).

[15] E. Witten, Quantum field theory and Jones' polynomial, Commun. Math. Phys. 121, 351-399 (1989).

Department of Physics, Graduate School of Science, University of Tokyo, Hongo 7-3-1, Bunkyo, Tokyo $113-$ 0033, JAPAN.

URL: http://gogh.phys.s.u-tokyo.ac.jp/ ${ }^{\sim}$ hikami/

E-mail address: hikami@phys.s.u-tokyo.ac.jp 